\title{
Le rôle des universités dans le développement des collections numériques québécoises
}

The Role of Universities in the Development of Numeric Collections of Québec Imprints

\section{El rol de las universidades en el desarrollo de las colecciones digitales quebequenses}

\section{Denis Boisvert}

Volume 54, numéro 3, juillet-septembre 2008

URI : https://id.erudit.org/iderudit/1029199ar

DOI : https://doi.org/10.7202/1029199ar

Aller au sommaire du numéro

Éditeur(s)

Association pour l'avancement des sciences et des techniques de la documentation (ASTED)

ISSN

0315-2340 (imprimé)

2291-8949 (numérique)

Découvrir la revue

Citer cet article

Boisvert, D. (2008). Le rôle des universités dans le développement des collections numériques québécoises. Documentation et bibliothèques, 54(3), 219-226. https://doi.org/10.7202/1029199ar
Résumé de l'article

Depuis l'émergence du Web au milieu des années 1990, le rôle et la place des documents traditionnels comme les livres et les périodiques sur support papier ont suscité de nombreuses réflexions chez les acteurs du développement des bibliothèques universitaires. En plus des échanges et des discussions établies dans le cadre de colloques ou de congrès, les intervenants dans les bibliothèques universitaires se sont également impliqués dans la réalisation de projets bien concrets de numérisation des collections.

Cet article porte essentiellement sur les enjeux associés au passage de l'analogique au numérique et de la bibliothèque traditionnelle à la bibliothèque virtuelle dans le milieu des bibliothèques universitaires québécoises depuis la fin des années 1990. C'est principalement en faisant le compte rendu des colloques et des journées de réflexion portant sur les problèmes associés à la diffusion et à la conservation des documents numériques que nous identifions quelques-uns des enjeux induits par la numérisation des collections dans ce milieu voué à l'enseignement et à la recherche. Une présentation des principaux projets de numérisation en cours est également faite à partir d'un document rédigé par Bibliothèque et Archives nationales du Québec (BAnQ) à ce sujet.
Tous droits réservés (c) Association pour l'avancement des sciences et des techniques de la documentation (ASTED), 2008
Ce document est protégé par la loi sur le droit d'auteur. L’utilisation des services d’Érudit (y compris la reproduction) est assujettie à sa politique d'utilisation que vous pouvez consulter en ligne.

https://apropos.erudit.org/fr/usagers/politique-dutilisation/ 


\title{
Le rôle des universités dans le développement des collections numériques québécoises
}

\author{
DENIS BOISVERT \\ Directeur, Service de la bibliothèque de l'UQAR \\ denis_boisvert@uqar.qc.ca
}

\section{RÉSUMÉ | ABSTRACTS | RESUMEN}

Depuis l'émergence du Web au milieu des années 1990, le rôle et la place des documents traditionnels comme les livres et les périodiques sur support papier ont suscité de nombreuses réflexions chez les acteurs du développement des bibliothèques universitaires. En plus des échanges et des discussions établies dans le cadre de colloques ou de congrès, les intervenants dans les bibliothèques universitaires se sont également impliqués dans la réalisation de projets bien concrets de numérisation des collections.

Cet article porte essentiellement sur les enjeux associés au passage de l'analogique* au numérique et de la bibliothèque traditionnelle à la bibliothèque virtuelle dans le milieu des bibliothèques universitaires québécoises depuis la fin des années 1990. C'est princip̀alement en faisant le compte rendu des colloques et des journées de réflexion portant sur les problèmes associés à la diffusion et à la conservation des documents numériques que nous identifions quelques-uns des enjeux induits par la numérisation des collections dans ce milieu voué à l'enseignement et à la recherche. Une présentation des principaux projets de numérisation en cours est également faite à partir d'un document rédigé par Bibliothèque et Archives nationales du Québec (BAnQ) à ce sujet.

\section{The Role of Universities in the Development of Numeric Collections of Québec Imprints}

With the rise of the Web in the mid-199os, the role and use of traditional documents, such as the paper versions of books and periodicals, has been the subject of some discussion on the part of university librarians. In addition to discussions held at meetings and conferences, librarians and other stakeholders are also involved in the scanning of collections.

This article focuses on the challenges associated with the transition from analogue ${ }^{* *}$ to numeric versions and from the traditional to the virtual library in the universities of Québec since the end of the 1990s. The proceedings of conferences and seminars that focused on the dissemination and conservation of scanned documents helped identify some of the challenges associated with this type of collection in university libraries. With the help of a document prepared by the Bibliothèque et Archives nationales du Québec (BAnQ), the author summarizes the major scanning projects currently in progress.
* Il s'agit de documents qui se trouvent sur support papier (imprimés, manuscrits, documents iconographiques, livres, revues...), mais aussi de documents audiovisuels et multimédias (enregistrements sonores, films, vidéos, cassettes..)

** We refer here to paper-based documents (books, manuscripts, photographs, periodicals) as well as audiovisual and multimedia documents (sound recordings, films, videos, etc.).

*** Se trata de documentos en papel (impresos, manuscritos, documentos iconográficos, libros, revistas, etc.) y también de documentos audiovisuales y multimedia (registros sonoros, películas, vídeos, casetes, etc.).
El rol de las universidades en el desarrollo de las colecciones digitales quebequenses

A partir del surgimiento de la Web a mediados de la década del '9o, el rol y el lugar de documentos tradicionales como los libros $y$ los periódicos, con respaldo en papel, han suscitado numerosas reflexiones entre los actores del desarrollo de bibliotecas universitarias. Además de los intercambios y de los debates que se desarrollan en el marco de coloquios o congresos, las personas involucradas en las bibliotecas universitarias se han visto igualmente implicadas en la realización de proyectos concretos destinados a digitalizar las colecciones.

El presente artículo aborda esencialmente los desafíos que implican el paso de lo analógico ${ }^{* * *}$ a lo digital y de la biblioteca tradicional a la biblioteca virtual en el medio de las bibliotecas universitarias quebequenses, desde fines de los años ' 90 . Es principalmente haciendo una reseña de los coloquios y jornadas de reflexión sobre los problemas vinculados a la difusión y a la conservación de documentos digitales que identificamos algunos de los desafíos inducidos por la digitalización de las colecciones en este medio dedicado al aprendizaje y a la investigación. Una presentación de los principales proyectos de digitalización en curso se ha implementado a partir de un documento redactado por la Biblioteca y los Archivos nacionales de Québec (BAnQ) sobre este tema.

\section{Introduction}

T ES BIBLIOTHÈQUES QUÉBÉCOISES, peu importe leur secteur d'activités, n'échappent pas au discours technologique dominant engendré par le processus de numérisation présentement en cours à l'échelle des grandes bibliothèques de ce monde. Le mouvement conduisant à l'intégration des documents multimédias et à l'élargissement de leur diffusion via Internet est irréversible. L'évolution rapide des technologies de l'information à laquelle nous assistons depuis une dizaine d'années a entraîné des changements majeurs dans une offre de services qui doit, de plus en plus, s'inscrire dans un nouveau paradigme, celui du partage de l'information à partir d'un environnement où les notions de territorialité et d'appartenance perdent de leur pertinence et de leur intérêt. De plus, la génération Google (UCL, 2008) qui est à la porte de nos institutions est habitée d'un sentiment de toute puissance informationnelle de nature à induire de nouveaux modèles dans l'organisation des services de bibliothèques (Ross, 2008). 


\section{La numérisation des collections : enjeux et contraintes sur le plan de la préservation et de la diffusion}

Les bibliothèques universitaires québécoises ont développé au cours des dernières années d'importantes collections de documents en format numérique. La majorité de ces collections sont acquises dans le cadre de consortiums regroupant d'importants réseaux de bibliothèques universitaires à l'échelle canadienne, dont le Réseau canadien de documentation pour la recherche $(\mathrm{RCDR})^{1}$ qui aura permis à l'ensemble de ces bibliothèques d'acquérir collectivement des ressources en format numérique dans le domaine de l'édition savante. Une partie importante des budgets d'acquisition est maintenant consacrée aux achats de produits électroniques en mode consortium.

L'un des enjeux majeurs de la numérisation est très certainement celui de la pérennité. La préservation de ces ressources électroniques n'est pas sans susciter des inquiétudes chez les bibliothécaires et les archivistes dans la mesure où les licences qui leur sont accordées portent essentiellement sur des droits d'accès à distance. La pérennité des accès à ces ressources documentaires en mode électronique hébergées sur des serveurs appartenant à des entreprises privées et commerciales est loin d'être assurée pour l'instant. De plus, les droits d'auteur et de propriété intellectuelle sont détenus par des éditeurs œuvrant pour la plupart des cas à l'étranger. Nous savons, par ailleurs, que les professeurs et les chercheurs du réseau des universités canadiennes publient la majorité de leurs articles dans des revues savantes détenues par des entreprises commerciales américaines et européennes.

L'accès à ces ressources numériques à distance est également assujetti aux applications de la Loi canadienne sur le droit d'auteur. Le matériel pédagogique qui est destiné à la production et à la diffusion de contenus en ligne dans le cadre d'activités d'enseignement et de recherche doit respecter le droit d'auteur canadien qui est très restrictif à cet égard. L'application de cette loi dans le secteur de l'enseignement a pour effet d'obliger les enseignants à obtenir toutes les autorisations requises auprès des auteurs ou des éditeurs avant d'utiliser ces contenus, même s'ils font partie des collections de leur bibliothèque d'appartenance.

La bibliothèque virtuelle offre de nombreux avantages : accès en ligne aux ressources numériques, portabilité de l'information à l'aide d'appareils de lecture évolués, élargissement de l'offre de biens et services multimédias intégrant une multitude de supports. Cependant les "désapports " (Pasquier, 2000) du numérique sont également nombreux en ce qui concerne plus spécifiquement l'application des principes sous-jacents à la mission des bibliothèques universitaires axée sur les notions de partage et de diffusion élargie des savoirs et des connaissances.

Les politiques de développement des collections numériques s'inscrivent dans le modèle dominant de protection des droits d'auteur, un modèle qui s'oppose à celui de la distribution éclatée de la propriété intellectuelle $^{2}$, du partage du patrimoine documentaire et de l'intégration de ces ressources à des outils technologiques. Cette nouvelle dimension impose aux gestionnaires des établissements universitaires de sérieuses contraintes en lien avec la mission des bibliothèques qui est de rendre le savoir accessible tout en optimisant l'utilisation des ressources qui leur sont accordées. Pour soutenir les universités dans l'organisation et la diffusion de la production intellectuelle des chercheurs, des professeurs et de tous les membres de la communauté universitaire au profit de la collectivité, le cadre législatif devrait, en évoquant la proposition formulée par Lawrence Lessing, favoriser des "modèles de développement économique et des cadres réglementaires qui s'harmonisent au partage et à la circulation du savoir permis par Internet »(Couture, 2008), ce que le régime actuel de droits d'auteur et de propriété intellectuelle ne permet pas. La politisation du patrimoine numérique et des technologies à la base de leur diffusion constitue le moyen le plus direct pour en assurer la démocratisation et la préservation pour les générations futures, mais aussi pour permettre aux universités de mieux répondre à leur mission d'enseignement et de recherche en assurant un partage équitable de leurs richesses patrimoniales.

\section{Les bibliothèques numériques à l'ère du Web 2.0 : un modèle axé sur l'usager et non plus sur les objets}

Les bibliothèques numériques s'inscrivent de plus en plus dans une dynamique de recherche établie par la configuration de portails de type Google et Amazon. Ces points d'entrée à la recherche sont élaborés à partir d'une architecture de type Web 2.0. Ainsi, comme nous le rappelle Mireille Lamouroux (2007), le projet de bibliothèque numérique européenne Europeana a été développé par la Bibliothèque nationale de France à l'aide d'outils open source. De la sorte, l'usager se voit offrir " un modèle centré sur les fonctions d'accès et d'utilisation des documents ainsi que sur l'utilisateur, et non plus sur les collections" (Lamouroux, 2007 : 390). Ce modèle de diffusion des connaissances favorise le partage de l'information tout en favorisant aussi l'étiquetage de portions de documents destinés à des usages dédiés. Il s'oppose au modèle dominant de diffusion des savoirs qui sont perçus comme des propriétés indivises.

Cette évolution des modes de recherche axés sur les technologies de l'information à l'ère du Web a pour 
effet de valoriser moins les objets que le sujet. Une transformation qui se reflète, entre autres, par l'intégration aux catalogues de bibliothèques de la recherche par facettes fondée sur le Web sémantique. Ces taxinomies de navigation donnent ainsi aux usagers un accès à des contenus enrichis à l'aide de métadonnées établies en fonction du type de ressource, du lieu, de la langue et des besoins spécifiques de l'usager, lui permettant d'obtenir des résultats de recherche à la hauteur de ses attentes. La majorité des systèmes de gestion de bibliothèques en place dans le réseau des bibliothèques universitaires québécoises ne sont pas configurés de manière à favoriser ce type de navigation. De plus, des modalités d'accès aux ressources d'origine numérique sont définies par des licences accordées par des entreprises privées à partir d'un modèle traditionnel de distribution de la richesse patrimoniale qui s'oppose au modèle alternatif axé sur l'utilisateur tel que favorisé par le Web 2.0.

Au cours des dernières années, les membres du sous-comité des bibliothèques de la Conférence des recteurs et des principaux des universités du Québec (CREPUQ) se sont penchés très sérieusement sur les défis et les enjeux de la numérisation. Afin d'illustrer l'intérêt des membres à cet égard et de bien saisir les enjeux associés à la numérisation du patrimoine documentaire dans le milieu des bibliothèques universitaires québécoises, nous allons faire état de l'avancement de la réflexion en cours et des démarches entreprises dans la préservation de la mémoire patrimoniale du Québec à partir de leur environnement en faisant référence à trois activités d'échanges et de discussions portant spécifiquement sur ces aspects. Deux activités ont pris la forme de journées de réflexion et une troisième celle d'un colloque.

\section{Journées de réflexion et d'échanges sur les défis de la numérisation au Québec (26 janvier et 11 octobre 2006)}

Dans le cadre d'un événement organisé conjointement par la CREPUQ et BAnQ en janvier 2006, les échanges et les discussions se sont cristallisés autour des défis soulevés par le développement et la création de collections numériques dans les diverses institutions concernées par le patrimoine documentaire québécois. Cette journée a regroupé une centaine de gestionnaires et de professionnels du milieu du patrimoine culturel et documentaire. Lors de cette journée de réflexion et d'échange, le point sur l'état des projets de numérisation au Québec a été fait. Les avenues possibles de collaboration et de coopération ont également fait l'objet de présentations à partir de modèles européens et canadiens. Les enjeux reliés à la mise en place d'actions et de politiques collectives pour renforcer la production d'informations numériques et pour favoriser la diffusion de sources d'information et le partage des ressources, de normes et d'expertises, ont suscité l'intérêt des parti-

\section{La nécessité de créer un patrimoine numérisé, afin d'en assurer la pérennité et l'accès élargi, a été reconnue par tous.}

$\alpha<\infty<\infty<\infty<\infty<\infty<\infty<\infty<\infty<\infty<\infty<\infty<\infty<\infty<\infty$

cipants. Des solutions ont été envisagées du côté des infrastructures communes, des partages d'expertises et de ressources, de partenariats régionaux et locaux, comme le mentionnait le professeur Jacques Grimard dans son bilan de la journée.

La nécessité de créer un patrimoine numérisé, afin d'en assurer la pérennité et l'accès élargi, a été reconnue par tous. Il a aussi été convenu d'adopter des politiques de numérisation de collections patrimoniales dans l'intérêt commun. Le rôle des bibliothèques nationales, et plus particulièrement celui de BAnQ, dans ce mouvement de numérisation s'est précisé lors de cette journée, en considérant la mission de sauvegarde et de préservation de la mémoire collective qui leur est accordée. Ainsi, BAnQ est appelée à jouer un rôle de soutien et de support dans le développement de politiques et de la mise sur pied de projets de numérisation en intervenant plus particulièrement au niveau de la définition des normes à adopter, en partageant son expertise et en offrant des sessions de formation.

Les interventions ont porté sur les cinq sujets suivants :

- état des lieux des fonds numérisés au Québec ${ }^{3}$;

- normes et standards de numérisation;

- numérisation et droits d'auteur;

- accès, difficultés de conservation et sécurité dans le domaine de la numérisation ;

- coordination des programmes de numérisation et infrastructures d'échange.

Au terme de la journée, il a été permis de constater que les universités québécoises sont très actives en matière de production et de diffusion de documents et de collections numériques. Pas moins d'une trentaine de projets et de réalisations figurent à l'état des lieux déposé par BAnQ. De plus, les membres ont démontré une réelle volonté d'assurer la pérennité des documents et des collections patrimoniales qui se retrouvent dans le réseau des bibliothèques universitaires aussi bien en format analogique qu'en format numérique. Ils ont également exprimé la volonté de se doter le plus rapidement possible d'une politique et d'un plan d'action national de numérisation établissant «les principes et les critères de choix des projets, et qui en déterminent le cadre d'allocation des ressources " (Grimard, 2006:9).

3. Nous reprenons en annexe la liste des projets de numérisation en cours de réalisation dans les universités québécoises. 


\section{C'est plutôt du côté des publications institutionnelles que des stratégies doivent être adoptées en vue de permettre aux universités de jouer efficacement leur rôle sur la scène de la numérisation.}

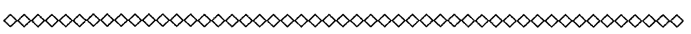

La journée du 11 octobre s'est déroulée dans le cadre d'une journée pré-congrès de l'ASTED organisée en collaboration avec BAnQ. Lors de cette rencontre qui faisait suite à la journée de réflexion du 26 janvier 2006, les participants ont pu assister à la présentation de divers projets et programmes de numérisation menés par des bibliothèques nationales, dont celles de la France et du Canada. Les projets européens Minerva ${ }^{4}$ et Michaels ont suscité beaucoup d'intérêt. Les conférences ont porté principalement sur les voies de concertation entre les différents partenaires animés par une volonté d'en arriver à une meilleure synergie dans la réalisation de projets de numérisation. Les dirigeants de Bibliothèque et Archives nationales du Canada (BAC) ont, à cette occasion, fait part d'une volonté d'adopter une stratégie canadienne à l'égard de la numérisation, à des fins de préservation et de diffusion du patrimoine documentaire canadien. Ils ont aussi fait l'annonce de la tenue d'un sommet national à Montebello en collaboration avec le Réseau canadien d'information sur le patrimoine (RCIP), l'Institut canadien de l'information scientifique et technique (ICIST), Alouette Canada et l'Université de l'Alberta. L'objectif de la réunion était de déterminer les éléments d'une stratégie nationale et les types de collaboration et d'investissements qui permettront de renforcer et de maintenir le patrimoine numérique au Canada.

Lors de la journée pré-colloque du 11 octobre, Claude Bonnelly a présenté trois modèles de gouvernance dans la création de projets nationaux de numérisation :

- un modèle centralisé ;

- un modèle coopératif à l'image du projet Initiative canadienne sur les bibliothèques numériques;

- un partenariat organisé et distribué à partir des modèles Alouette Canada et European Digital Library.

Le modèle coopératif décentralisé et articulé autour de BAnQ comme chef de file a retenu l'attention des membres. Une table de concertation, regroupant des pôles régionaux, a aussi été envisagée afin de favoriser l'adoption de politiques et de programmes répondant aux besoins de l'ensemble de la population québécoise.

\section{Session de réflexion du 30 novembre 2006}

Cette session à l'intention des directeurs des bibliothèques universitaires québécoises concernait le suivi à apporter aux journées d'information du 26 janvier 2006 et du 11 octobre 2006 portant sur les problématiques de la numérisation de documents textuels et non textuels d'intérêt pour les bibliothèques québécoises.

Pour cette rencontre, tenue dans les locaux de la Grande bibliothèque, les participants avaient à définir leur position à l'égard de la problématique suivante : comment les bibliothèques universitaires conçoiventelles leur éventuelle contribution au soutien de la constitution de collections de documents numériques? Il s'agissait davantage d'un exercice de réflexion axé sur la prospective en vue de dégager des pistes d'actions à explorer.

Lors de cette journée de réflexion, les participants ont encore une fois insisté sur la nécessité de rendre démocratiquement accessible un vaste patrimoine documentaire en format numérique dans le respect de la mission d'enseignement et de recherche des universités québécoises. Trois types de documents patrimoniaux ont été identifiés en fonction de leur attribution d'origine : national, international et institutionnel. En ce qui concerne les documents patrimoniaux à caractère national, il a été entendu qu'il appartient à BAnQ d'en assurer la préservation et la diffusion en mode numérique en lien avec leur mission. Pour les documents de type international, des institutions supranationales ont le mandat d'en établir la préservation et la diffusion en mode électronique. C'est plutôt du côté des publications institutionnelles que des stratégies doivent être adoptées en vue de permettre aux universités de jouer efficacement leur rôle sur la scène de la numérisation. Des milliers de revues savantes sont directement alimentées par les publications de chercheurs, de professeurs ou d'étudiants aux études supérieures. Chaque année, il se publie dans le réseau des universités canadiennes environ 30 ooo thèses de maîtrise et de doctorat. Ces documents sont acquis et traités par Thèses Canada ${ }^{6}$ qui en assure le prêt et la diffusion par le biais d'UMI Dissertations Publishing. Dans le réseau des universités québécoises, une partie seulement de ces ressources sont accessibles sur le Web.

La gestion des métadonnées descriptives liées aux documents d'origine numérique ou encore aux documents analogiques convertis au format numérique pose aux bibliothécaires de sérieux défis en lien avec les systèmes de gestion documentaire en place. Les normes et les pratiques ne sont pas encore uniformes de sorte que la mise en commun des ressources patrimoniales et leur intégration dans un portail unique ne peuvent se 
réaliser facilement. La diffusion élargie de ces données en mode Web n'est pas encore assurée.

Les gestionnaires de bibliothèques se sont également penchés sur les enjeux et les défis que pose le respect du droit d'auteur et de la propriété intellectuelle s'appliquant aux documents numériques en milieu académique. Le cadre juridique est très limitatif à cet égard.

$\mathrm{Au}$ terme de la rencontre, deux types de documents ont été identifiés par les participants pour leur intérêt patrimonial en lien avec des projets de numérisation : les thèses et les banques d'images.

En ce qui concerne les images, il avait également été suggéré de prendre en compte la problématique de la conservation des ressources documentaires intégrées aux collections audiovisuelles et multimédias. Il est reconnu que les documents audiovisuels et multimédias font partie intégrante de notre patrimoine documentaire. À ce sujet, mentionnons que les enjeux associés à la conservation des documents audiovisuels et multimédias, et notamment les questions éthiques, font l'objet des préoccupations des membres des sections Préservation et Conservation, Technologies de l'Information, Construction et Équipements de l'IFLA (Giannattasio, 1998). Au cours des années, les supports changent et les équipements qui en permettent l'usage deviennent de plus en plus défectueux. Ce mouvement s'inscrit dans un contexte de constante ébullition technique et industrielle. À côté des possibilités élargies du Web, le besoin d'accéder à des images en format électronique se fait de plus en plus sentir. Les champs d'application dans le domaine de l'enseignement et de la recherche demeurent ouverts et très intéressants. Des projets en émergence, tels que Digital Libraries : Resources and projects (IFLA) ; The Digital Library Federation (DLF) et Directory of Digitized Collections (UNESCO) représentent des applications d'intérêt pour les bibliothèques universitaires québécoises.

La production de thèses et de mémoires disponibles uniquement en mode numérique doit également faire l'objet d'un projet collectif. De ce côté, certaines universités ont déjà réalisé des projets d'intérêt. Ainsi, à la suite à un vaste programme de numérisation de ses documents rétrospectifs, le Service de la bibliothèque de l'UQAC, donne accès au texte intégral de tous les mémoires et thèses produits dans cette institution depuis sa fondation grâce à son Portail des mémoires et thèses 7. Par ailleurs, dès 2007, l'Université Laval a rendu obligatoire le dépôt de mémoires et de thèses en format électronique. À l'Université de Montréal, une formation est accordée à tous les doctorants pour qu'ils puissent rédiger leur thèse en format électronique à partir d'une définition préétablie de métadonnées. Aussi, en collaboration avec la Faculté des études supérieures et postdoctorales (FESP), un projet de numérisation des thèses

7. http://theses.uqac.ca/a-propos.php
Deux types de documents ont été

identifiés par les participants pour

leur intérêt patrimonial en lien avec

des projets de numérisation: les

thèses et les banques d'images.

00000000000000000000000000000000000000000

déposées par le passé en format papier est présentement à l'étude.

\section{Modèle de gestion}

Il a été fortement suggéré de constituer un registre de numérisation dans le réseau des bibliothèques universitaires québécoises afin de recenser les projets en cours, mais aussi de les faire connaître au plus grand nombre possible d'acteurs concernés. Bibliothèques et Archives Canada (BAC) a déjà mis sur pied un tel registre qui prend la forme d'un site Web qui s'intitule: Répertoire des projets canadiens de numérisation ${ }^{8}$. Par ailleurs, BAnQ entend également créer un tel répertoire dans le cadre de son projet intitulé Initiative québécoise en matière de numérisation du patrimoine documentaire pour le Secrétariat général.

En ce qui concerne la coordination et la mise à jour d'un tel registre, il a été convenu d'en confier la responsabilité au groupe de travail BRVQ (Bibliothèque de recherche virtuelle du Québec), relevant du sous-comité des bibliothèques de la CREPUQ. Les directeurs ont convenu de confier au groupe de travail le mandat d'élaborer un plan stratégique en matière de numérisation, de façon à contribuer au soutien et au développement de collections de documents numériques d'intérêt pour le réseau des bibliothèques universitaires québécoises.

\section{Journée de réflexion du 9 mai 2008, organisée par le sous-comité des bibliothèques de la CREPUQ}

Une autre journée de réflexion à l'intention des directeurs des bibliothèques universitaires québécoises a eu lieu le 9 mai 2008. Elle portait sur la reconceptualisation des services des bibliothèques universitaires québécoises. Cette journée de réflexion a été organisée par le sous-comité des bibliothèques de la CREPUQ. Les personnes ressources invitées, Mme Alma Swan ${ }^{9}$ et $\mathrm{M}$. David Lewis ${ }^{10}$, ont mis en perspective le rôle des bibliothèques universitaires en lien avec l'évolution des nouvelles technologies de l'information au cours des prochaines années. Ces conférenciers ont sensibilisé les gestionnaires de bibliothèques au fait qu'ils devront

8. http://www.collectionscanada.ca/initiatives/index-f.html

9. Directrice, Key Perspectives Ltd (Grande-Bretagne)

10. Dean of the University Library, Purdue University 
La prédominance du numérique sur l'imprimé ou les documents traditionnels obligera les bibliothèques universitaires à revoir l'ensemble de leurs activités à l'intérieur de cette nouvelle réalité.

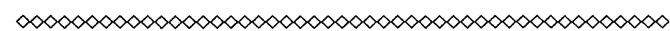

de plus en plus intégrer des programmes de numérisation des collections dans leurs stratégies de développement afin de répondre aux besoins et aux attentes des étudiants de la génération Google. La place de la bibliothèque dans l'espace universitaire sans l'adoption de stratégies axées sur la migration de l'imprimé à l'électronique est, selon ces chercheurs, dangereusement compromise.

\section{Journée de réflexion du 28 mai 2008 organisée par le comité des bibliothèques de l'Université du Québec}

Le 28 mai 2008, le comité des bibliothèques de l'Université du Québec tenait à l'Université du Québec à Montréal (UQAM) une journée de réflexion sur l'impact des changements technologiques sur l'offre de services, en lien avec le rôle des bibliothèques dans leur mission de soutien à l'enseignement et à la recherche. Il a été reconnu par tous les participants à cette journée que la prédominance du numérique sur l'imprimé ou les documents traditionnels obligera les bibliothèques universitaires à revoir l'ensemble de leurs activités à l'intérieur de cette nouvelle réalité. Un constat majeur a dominé l'ensemble des discussions : le changement profond engendré par l'arrivée du numérique dans le monde de l'information. Les participants se sont également interrogés sur le rôle et l'efficacité des moteurs de recherche intégrés à leur nouveau système de gestion de bibliothèque (Aleph d'ExLIbris) par rapport à un outil de navigation aussi puissant que Google qui est devenu la porte d'accès quasi unique à Internet. Un autre questionnement a aussi été soulevé à l'égard de l'avenir de la bibliothèque numérique francophone : veut-on une bibliothèque pour les chercheurs ou pour les étudiants de premier cycle ? Les collections numériques dans le monde universitaire sont principalement disponibles en langue anglaise et, en contrepartie, les collections de livres électroniques en langue française sont, à toutes fins pratiques, inexistantes. Cette réalité a un impact majeur sur le développement des collections numériques dans le milieu des bibliothèques francophones du réseau universitaire québécois, qui touche plus particulièrement les constituantes du réseau UQ implantées en région.

Il nous est donc permis de constater que les responsables des bibliothèques universitaires québécoises s'inscrivent dans une dynamique de valorisation et de diffusion des collections numériques qui se retrouvent dans l'ensemble du réseau. Ils sont également conscients des enjeux et des défis que pose l'adoption de projets de numérisation de documents patrimoniaux. La mise sur pied d'un chantier de numérisation requiert une grande rigueur méthodologique, des ressources humaines et financières, l'adoption d'un plan d'action ordonné, le recours à des normes établies, mais avant tout une définition des politiques en matière de numérisation de ressources documentaires en vue de favoriser la concertation et le partage de ces richesses documentaires qui sont surtout des outils de développement social, économique et culturel. $\odot$

\section{Sources consultées}

Cavalcante, Lidia Eugenia. 2007. La mémoire patrimoniale du Québec: préservation, politiques et actions pour la numérisation des collections patrimoniales. Documentation et Bibliothèques, vol. $53, \mathrm{n}^{\circ} 2: 85-101$.

Couture, Stéphane. 2008. Gilberto Gil : politiser la culture numérique, Le Devoir, 27 février 2008, Section Alternatives, p. 6.

Giannattasio, Isabelle. 1998. Round Table on Audiovisual ans Multimedia, Conservation des documents audiovisuels et multimédias : Questions éthiques. International Federation of Library and Institutions (IFLA).

Grimard, Jacques. 2006. Le patrimoine documentaire du Québec à l'heure du virage numérique. À rayons ouverts: Chroniques de la Bibliothèque nationale du Québec, $\mathrm{n}^{\circ} 68:$ : 8-10.

Lamouroux, Mireille. 2007. Les publics des bibliothèques. Documentaliste - Sciences de l'information, vol. 44, $\mathrm{n}^{\circ} 6: 389-391$.

Pasquier, Florent. 2000. Du support analogique au document informatisé pour l'apprentissage : les « désapports » de la numérisation. La revue de l'Epi, $\mathrm{n}^{\circ} 100: 109-116$.

Les ressources documentaires numériques au Québec: Bibliothèques - Musées - Universités : un aperçu. 2006. Montréal : Bibliothèques et Archives nationales du Québec.

Ross, Lyman. 2008. The Library is Dead, Long Live the Library! The Practice of Academic librarianship and the Digital revolution. The Journal of Academic Librarianship, vol, 34, $\mathrm{n}^{\circ} 2$ : 145-152. 
University College of London (UCL). 2008. Information behaviour of the researcher of the future: a CIBER briefing paper. London, UCL.

\section{Annexe 1 : Projets de numérisation en cours dans les universités québécoises}

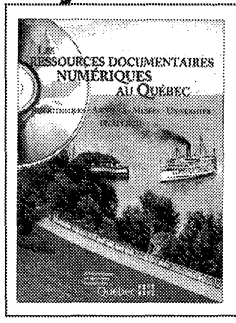

Le texte qui suit est extrait de : Les ressources documentaires numériques au Québec : Bibliothèques - Musées - Universités : un aperçu. Montréal : Bibliothèques et Archives nationales du Québec, 2006 : 3-6. Le document est accessible en version numérique à http://collections.banq.qc.ca/ark:/52327/ bs58518

\section{Le monde universitaire}

Les universités québécoises jouent un rôle très actif en matière de production et de diffusion de documents numériques. Aux initiatives purement locales s'ajoutent des projets qui concernent toutes les universités québécoises, certains s'insérant dans des initiatives qui touchent l'ensemble des universités canadiennes.

L'Université de Montréal, l'Université Laval et l'Université de Montréal $[s i c]^{11}$ ont formé une société sans but lucratif, Érudit (http://www.erudit.org/), qui vise à rendre disponible sous forme numérique la production savante québécoise. Érudit a produit et diffuse plus de 18 ooo articles provenant de 48 revues savantes, des livres (13 titres), des thèses et autres documents et données (près de 1 ooo titres). Des travaux de numérisation rétrospective sont prévus pour porter à près de 150000 le nombre des articles de périodiques disponibles, de même que le lancement d'un service d'édition d'actes de colloques et de congrès.

La chaîne de production Érudit a été adoptée en France par le projet Persée (http://www.persee.fr/), sur les revues scientifiques en sciences humaines et sociales ainsi que par le Centre d'édition numérique scientifique (CENS) du Centre national de la recherche scientifique (CNRS), ce qui assure une interopérabilité entre les productions québécoise et française.

L'Université de Montréal participe au projet de numérisation des thèses de Bibliothèque et Archives Canada. La Direction des bibliothèques prévoit se doter d'une infrastructure de gestion des projets de numérisation de documents et de collections. En 2004, un projet pilote de numérisation de diapositives en architecture a été réalisé à la Bibliothèque d'aménagement. D'autres projets sont envisagés : cartes géographiques, affiches de guerre, partitions musicales et documents du Service des livres rares et des collections spéciales.
Par ailleurs, la Direction des bibliothèques gère aussi un dépôt institutionnel (documents et données de recherche, prépublications et publications). En service depuis mars 2005, le dépôt est accessible à l'adresse suivante : papyrus.bib.umontreal.ca.

La Division des archives de l'Université de Montréal a numérisé plus de 2000 documents qui sont diffusés sur Internet (http://www.archiv.umontreal.ca/docnum. htm).

L'Université McGill a réalisé plus de 50 projets de numérisation depuis 1997 (art et architecture, histoire de la médecine, sciences et ingénierie, etc.). Les documents sont accessibles à l'adresse http://www.mcgill.ca/ $\mathrm{dcp} /$. Parmi les initiatives récentes, figure la numérisation de 3000 enregistrements de jazz des années 1940 et 1950 (http://coltrane.music.mcgill.ca/mapp/index. html).

À l'Université Concordia, la bibliothèque est en voie de numériser la collection $\mathrm{S}$. A. Rochlin relative aux organisations politiques et ouvrières d'Afrique du Sud. L'Université participe aussi au programme de numérisation des thèses de Bibliothèque et Archives Canada.

L'Université du Québec à Montréal (UQAM) est membre d'Érudit et participe aussi au programme de numérisation des thèses de Bibliothèque et Archives Canada. Une réflexion est en cours sur la constitution d'une banque d'images numérisées et d'une banque d'objets d'apprentissage. Depuis 1997, le Service des archives et de gestion des documents possède un laboratoire de numérisation, qui réalise des travaux autant pour les documents administratifs (procès-verbaux, sentences arbitrales, comptes à payer, etc.) que pour les archives historiques, institutionnelles ou privées.

La bibliothèque de l'Université du Québec à Chicoutimi (UQAC) est engagée dans deux projets de numérisation importants :

- les Classiques des sciences sociales (http:// classiques.uqac.ca/) un ensemble de quelque 2200 textes regroupés sous sept collections, y compris des ouvrages récents dont les auteurs ont autorisé la diffusion. L'objectif est de faire de ce site une des grandes sources pour l'accès à la documentation de langue française en sciences sociales.

- la collection Thèses et mémoires de l'UQAC vise plus de 1400 documents, accessibles à l'adresse suivante : theses.uqac.ca

L'UQAC soutient aussi son propre dépôt institutionnel à partir d'un logiciel développé localement (SOURCIER) pour les documents de caractère universitaire et d'intérêt régional (sdeir1. uqac.ca).

L'Université du Québec à Rimouski (UQAR) a entrepris un programme de numérisation de ressources documentaires d'intérêt régional : journaux, livres et brochures, fonds d'archives. Un plan d'action en vue de

11. Il faudrait lire plutôt l'Université du Québec à Montréal. 
numériser les thèses et mémoires a également été adopté par la direction de l'Université.

L'Université du Québec à Trois-Rivières (UQTR) participe au programme Thèses Canada de Bibliothèque et Archives Canada. Le Département des sciences du loisir et de la communication sociale produit la revue Loisir et société, disponible via Érudit. L’Observatoire québécois du loisir et son réseau de partenaires développent aussi la Bibliothèque électronique en loisir (BEL : https ://www2.uqtr.ca/archimede_bel) (politiques, plans d'action et rapports de recherche ou de consultation).

L'Université Laval a lancé un grand nombre de projets de numérisation. Elle a également accumulé une vaste expérience dans ce domaine et sur les technologies associées : reconnaissance optique de caractères et gestion de bases de données appuyées de plus en plus sur des outils informatiques libres de droits. Parmi les réalisations marquantes de la Bibliothèque, on trouve :

- Notre mémoire en ligne / Early Canadiana Online (http://www.canadiana.org/), projet qui résulte d'une collaboration entre l'Université de Toronto et l'Université Laval. Entre 1997 et 2000, les deux institutions ont numérisé 500000 pages de documents sur microfiches de l'Institut canadien de micro-reproductions historiques (ICMH), devenu depuis Canadiana.org.

- Nos Racines / Our Roots (http://www.nosracines. $\mathrm{ca} /$ ) est un projet réalisé en collaboration avec l'Université de Calgary (Alberta) et l'Université Laval. Il porte sur les documents d'intérêt concernant l'histoire locale et régionale partout au Canada. Environ 600 ooo pages provenant de plus de 4 ooo livres ou brochures sont disponibles à ce jour.

- Les thèses et mémoires électroniques (http:// www.theses.ulaval.ca/) se conforment aux normes technologiques qui permettent notamment à Thèses Canada d'en assurer le repérage automatique. L'Université Laval a incidemment été choisie pour accueillir le congrès international ETD 2006, sur les mémoires et thèses électroniques.

- Archimède (archimede.bibl.ulaval.ca) gère le système de dépôt institutionnel qui couvre les publications des facultés et groupes de recherche de l'Université. C’est un logiciel libre, inspiré du modèle Dspace du MIT.

- D'autres projets de numérisation ont aussi été réalisés par d'autres unités de l'Université Laval, notamment le Dictionnaire biographique $d u$ Canada (http://www.biographi.ca/FR/) réalisé en collaboration avec l'Université de Toronto.
Plusieurs projets de numérisation sont en cours à l'Université de Sherbrooke :

- La Chaire de recherche en histoire du livre et de l'édition vise la numérisation de tous les types de documents relatifs à l'histoire du livre et de l'édition au Québec : articles et livres sur le livre, archives d'éditeur et d'auteur, photos, catalogues, rapports de recherche, mémoires, thèses et rapports, etc.

- Les groupes de recherche CATIF et FRANQUS (Groupe de recherche sur le français au Québec : usage et standard) numérisent des documents en texte intégral pour étudier les usages linguistiques québécois (franqus.usherbrooke.ca/travaux.html).

- Le Service des archives projette la mise en place d'un programme de numérisation des archives inactives institutionnelles et privées dont il assure la conservation et la diffusion. Ainsi, le Service a réalisé la numérisation du fonds Anne Hébert, en vue d'offrir aux chercheurs l'accès sur place aux images des documents uniques de ce fonds.

À l'Université Bishop's, plusieurs projets sont à l'étude ou en cours :

- Le Bureau des collections spéciales et collections d'archives de la bibliothèque numérise divers documents officiels dont les procès-verbaux du Sénat de l'Université.

- Une variété de documents, y compris des photographies provenant de fonds d'archives privées, sont régulièrement numérisés pour les chercheurs. Certains de ces documents sont diffusés sur le site Internet de l'Université (http://www. ubishops.ca/), notamment dans sa section sur l'histoire de l'Université.

- Le Bureau de la diathèque de l'Université a récemment mis en place un projet de numérisation de sa collection de diapositives dont les droits d'auteur ont été libérés.

- La bibliothèque examine la possibilité de numériser les pages couverture de sa collection de partitions musicales. Ces images apparaîtraient dans le catalogue en ligne, liées aux descriptions bibliographiques. La bibliothèque élabore également un projet de numérisation des archives $\mathrm{du}$ fonds Festival Lennoxville (photographies, documents, manuscrits, etc.). 